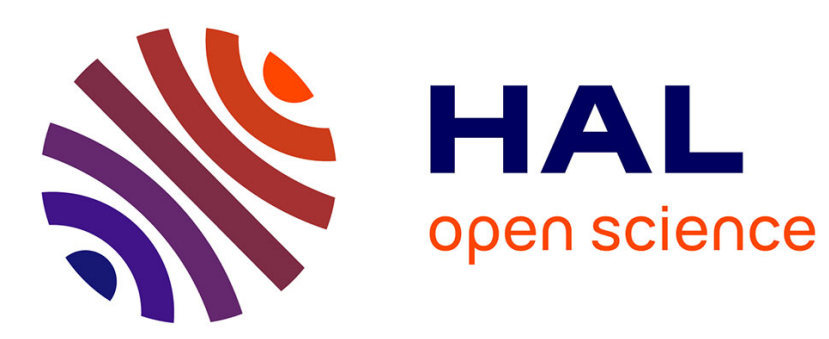

\title{
Knowledge of Domain Effects in Problem Representation: The Case of Tower of Hanoi Isomorphs
}

Evelyne Clément, Jean-François Richard

\section{To cite this version:}

Evelyne Clément, Jean-François Richard. Knowledge of Domain Effects in Problem Representation: The Case of Tower of Hanoi Isomorphs. Thinking and Reasoning, 1997, 3, pp.133 - 157. hal-02004921

\section{HAL Id: hal-02004921 https://hal-cyu.archives-ouvertes.fr/hal-02004921}

Submitted on 12 Feb 2019

HAL is a multi-disciplinary open access archive for the deposit and dissemination of scientific research documents, whether they are published or not. The documents may come from teaching and research institutions in France or abroad, or from public or private research centers.
L'archive ouverte pluridisciplinaire HAL, est destinée au dépôt et à la diffusion de documents scientifiques de niveau recherche, publiés ou non, émanant des établissements d'enseignement et de recherche français ou étrangers, des laboratoires publics ou privés. 
Knowledge of domain effects in problem representation: the case of Tower of Hanoi isomorphs

Evelyne Clément* and Jean-François Richard**

*UFR de Psychologie. Université de Rouen

Rue Lavoisier.

F 76821 Mont Saint Aignan Cedex, France

**UFR de Psychologie. Université de Paris 8

2 rue de la Liberté

F.93526 Saint Denis Cedex 02, France

Tel: 33149406479

Fax: 33149406754 
E. Clément and J.F. Richard

\begin{abstract}
Differences in difficulty between isomorphs of the Tower of Hanoi, are generally explained in terms of differences in processing loads required by the different versions (Kotovsky and Fallside, 1989). Our claim is that the general knowledge about an action, activated by the context, is what guides the elaboration of problem representation . To test this hypothesis, we manipulated the context using 4 isomorphs. The results support the hypothesis: the selection of the adequate point of view on the action depends on the context, and is a crucial step in the elaboration of problem representation. The more difficult versions are those which require abandoning the first point of view and selecting a new one.
\end{abstract}


A puzzling question in problem solving is why isomorphic problems vary so much in difficulty despite the fact that they share the same problem space and, consequently, are equivalent as to the size of the problem space that must be explored. A related result is that there is usually little transfer across problems and the transfer effects are often asymmetrical. Such results have been obtained with isomorphs of the missionaries and cannibal problem (Reed, Ernst and Banerji, 1974) and with Duncker's radiation problem (Gick and Holyoak, 1980, 1983). As a rule, the analogy is hardly perceived, unless a hint is given that the solution of the first problem might have something to do with the solution of the second (Reed, Ernst and Banerji, 1974, Gick and Holyoak, 1980) or unless several isomorphs have already been solved prior to the test problem (Gick and Holyoak, 1983).

The most extensive studies have been conducted on isomorphic versions of the Tower of Hanoi problem and the well-known monster problems (Simon and Hayes, 1976; Hayes and Simon, 1977; Kotovsky, Hayes and Simon, 1985; Kotovsky and Fallside, 1989). These problems are of two kinds: "move problems", in which the "operator" (the action) consists of changing the place of objects, and "change problems", in which the operator consists of changing the size of the objects. In move problems, three globes (large, medium, small) held by three monsters differing in size are to be transferred from monster to monster until each monster has the appropriatesized globe. In change problems, each monster holds a globe of a given size and the size of each globe may be changed. There are conditions allowing these changes, which are equivalent in both types of problems. A common rule is that only one globe may be changed (or transferred) at a time. The other two rules are:

Rule 2.

Move: "If a monster is holding two globes, only the larger of the two may be transferred." 
Change: "If two globes have the same size, only the globe held by the larger monster may be changed."

Rule 3.

Move: "A globe may not be transferred to a monster who is holding a larger globe."

Change: "A globe may not be changed to the same size as the globe of a larger monster" (from Kotovsky, Hayes and Simon, 1985).

As can be seen, in the move problems, the conditions for transferring a globe concern the size of globes in the same place (held by the same monster); in change problems, the conditions for changing the size of a globe concern the size of monsters holding globes of the same size.

Kotovsky, Hayes, and Simon (1985) investigated several possible causes of differences in difficulty: (i) the nature of the operator used in the various versions (change vs. move), (ii) differences in the degree of consistency between the problem rules and world knowledge (Acrobats versus Reverse Acrobats), and (iii) the way the problem is presented (the concrete Tower of Hanoi versus paper and pencil in isomorphic versions). Differences of sixteen times the solution time were obtained between the hardest problem (change problem in paper and pencil format) and the easiest problem (the classical 3-disk Tower of Hanoi problem). The authors claim that the greater difficulty of change problems is due to the difference in memory load required by the processing of the tasks: (i) in move problems, testing moves for legality requires comparing objects at a single locus, so that the subject can produce a spatial representation that reduces the memory load, while in change problems the objects to be compared are at two different loci, (ii) versions having rules which are consistent with real-world knowledge reduce the memory load involved in remembering the conditions of application of the operator, and (iii) the concrete problem presentation in which rule information is incorporated (the concrete Tower of Hanoi) reduces the memory load in comparison with a purely mental representation. The results are 
summarized in the following terms by Kotovsky and Fallside (1989): "The processing loads of the different move operators could be ranked in terms of the number of entities (globes, monster loci) that had to be imaged in order to test the legality of a move. This ranking was predictive of both the difficulty of making individual moves and also of overall problem difficulty." (p72-73).

A second main conclusion of this study was that problem solving consists of two phases: an exploratory phase with no apparent planning, during which the subject does not get nearer to the goal and often reverts to the initial state, and a "final path" phase, in which the moves are fast, relatively error free, and exhibit planning. The difference between isomorphic problems lies only in the exploratory phase, as there is almost no difference between isomorphs as regards the "final path" phase. These results show that what subjects learn during the exploratory phase is to practice and automate move making: they learn to understand and apply the operator.

In a further study, Kotovsky and Fallside (1989) showed that transfer has the same effect as practice: the locus of transfer is the exploratory phase and the determinant of transfer is not the similarity of the stimuli but the similarity of the internal representation of the problem. Actually two problems were used which involve the same stimuli but with different "embodiments" (or cover stories): the transfer is much more complete when the second problem belongs to the same context (can be interpreted as a change in position or a change in size) and is not affected by a change in the stimulus.

The present study was aimed at further exploring the processes involved in understanding the operator in the various contexts provided by Tower of Hanoi isomorphs. Why is it so much more lengthy to understand a change in size than a change in position? The memory load difference produced by the operations needed to check the legality of a change compared to a move is supported by strong arguments, as shown by Kotovsky, Hayes, and Simon (1985). But there is another possible 
explanation for the effects of the factors Kotovsky, Hayes, and Simon (1985) called "rule learning", "rule application", and "memory load". The reason why rule learning is much more difficult in change problems than in move problems may lie in the fact that the appropriate interpretation of the operator is obvious in Tower of Hanoi problems, being suggested by the context, while the immediate interpretation evoked by the context of a size change problem is irrelevant to the problem and has to be modified in order to meet the problem requirements. We shall now explain this hypothesis in greater detail.

As a rule, any action may be considered from two points of view (Richard, 1990): that of the result of the action, which is a change of state, and that of the way it is actually carried out, i.e. the process of transformation leading from the initial state to the final state. We shall call these two aspects the "change-of-state viewpoint" and the "transformation viewpoint". When the state-change viewpoint is taken, the global action is considered only as a change of state (the object is no longer there, it is here). The action may be easily decomposed into two subgoals: getting out of one state and getting into a new one. When the transformation viewpoint is taken instead, the global action is seen as a transition process from one state to another. It is not decomposed into subgoals referring to the initial and final state, since the emphasis is on the transition between states.

In Tower of Hanoi problems, it is relevant to consider the change in state produced by the operator and not the transformation itself, for the following reason: if a move is conceived of as removing an object from one place and putting it in another, rule 2 and rule 3 of the instructions are easily understood as the conditions for the action of removing the disk and for the action of putting it elsewhere, respectively. The condition for removing a disk from a given place is that it be the smallest in the pile (and therefore on the top) and the condition for being able to put it elsewhere is that there be no smaller disk or no disk at all in that place. 
In the same way, changing the size of an object should be interpreted as follows: the object loses its current size and takes on a new one, as if by means of a magic wand. As in text processing, the effect of the cut and paste commands is to remove a text from one place and insert it in another. In this case, rules 2 and 3 may be interpreted as the conditions for each of these component actions: the condition for losing a size is that there be no globe of the same initial size held by a larger monster, and the condition for taking on a new size is that there be no globe of that new size held by a larger monster. It then becomes easy to transform these conditions into prerequisites and subgoals: to change the current size of a given globe, first change the size of the other globes of the same size held by larger monsters (if there are such globes); to give a new size to a globe, change the size of the globes held by larger monsters that are already that new size, if there are any. There are some observations which suggest that in the change case, the operator is interpreted as a transformation process rather than as a mere change of state: in such problems, a fair proportion of subjects understand that it is legal to change a size to a size near it and prohibited to change a size directly from small to large or from large to small. In an unpublished study, Clement (1990) showed through the analysis of verbal protocols that some subjects state explicitly that it is prohibited to directly change a size from large to small or vice versa, and that it is necessary to go from small to medium and then from medium to large. In their interpretation, change of size is seen as a continuous process like growth. This misconception may be very persistent. It is not observed in adults solving Tower of Hanoi problems. It may appear in some adults in other problem-solving situations, but disappears very quickly. However, this misconception was observed in children in a study by Richard and Poitrenaud (1988). A substantial proportion of the children aged 6-7 years refused to move a disk from left to right (or right to left) if there was a smaller disk in the middle (the disks did not have to be put on a peg but only laid down in an empty place or on another disk): a number of children thought that going from left 
to right implied going through the middle. In this case, the three places, lined up in a row, are interpreted as a road: a move is conceived of as a move in the plane and not as a move in the third dimension, as it should be. This is consistent with the fact that at this age, "moving" has the prototypical meaning of moving on the ground (see Bernicot, 1981).

In our opinion, these misconceptions are due to the fact that the operator is grasped from the standpoint of the transformation being performed, i.e. from the point of view of the process that is taking place. What is needed is a change in the way of coding what a move is. Instead of considering a move as transition process, which is the usual way (at least in the change of size operator and in the move operator for children), a move has to be recoded as a mere change of location, without considering the path followed to realize the action. Coding a move as a change of state is more abstract than coding it as a transformation process.

We think that the effects of rule learning, rule application, and spatial memory load proposed by Kotovsky, Hayes, and Simon (1985) to account for differences among isomorphs are due to the same factor: the building of an adequate representation of the operator. In Tower of Hanoi problems the action of moving is readily interpreted as decomposable into two subactions corresponding to leaving the old state and entering the new one, while in change-of-size problems, the size change is understood as an atomic, non-decomposable action. This is explained by the assumption that the Tower of Hanoi problem emphasizes the state-change point of view, while the change-of-size problem emphasizes the transformation-process point of view.

In other words, we agree with Kotovsky, Hayes, and Simon (1985) and Kotovsky and Fallside (1989) when they say that the differences between isomorphs lie in move learning, but we disagree with their conception of move learning. Move learning is understand by these authors as a process of move automation, allowing for a reduction in memory load. We think that move learning is a semantic process, 
consisting of discovering the appropriate interpretation of the operator and building the appropriate problem space.

To test this hypothesis about the role of the point of view, it is necessary to show that a move problem in which the transformation aspect is made more salient than the state-change aspect, is more difficult, and conversely, that a change problem in which the change of state is made more salient, is easier. Moreover, if we assume that the only difference between a move problem and a change problem is the emphasis on the point of view, we shall predict that a move problem in which the transformation point of view is made more salient is as difficult as a size-change problem, and equivalently, that a change problem in which the state-change point of view is made more salient should be as easy as a Tower of Hanoi problem.

This hypothesis was tested in our first experiment using four isomorphs: two move problems, where the operator was "move an object from one place to another," and two change problems in which the operator was "change the size of an object that stays in the same place." The four problems are as follows:

Move problems. The version which emphasizes the change-of-state viewpoint is the classical Tower of Hanoi problem, since subjects immediately interpret the action of moving a disk as removing a disk from its current place and putting it in a new one.

In order to make the transformation point of view more salient, we chose a problem called "The Lift" where people have to be transported from floor to floor. In our everyday environment, we know that one has to go through all intermediate floors when taking a lift from one floor to another. This knowledge should influence problem solving behavior by inducing an inadequate problem representation.

Change problems. In order to emphasize the change-of-state point of view, we used a problem in which the operator was "change the value of a counter". The counter had three possible values, 1, 2, and 3, and could be turned clockwise or counterclockwise so that it was possible to directly change 1 into 3 or vice versa without going through 2 . 
The version emphasizing the transformation point of view was similar to the monster problem. Three blocks of different sizes (small, medium, large) were placed in three different places (left, middle, right) each held by a person.

Kotovsky and Simon's definition of isomorphism was used here: our problems are isomorphic because "the graph of one problem can be mapped onto the graph of the other, with nodes and links corresponding one to one" (Kotovsky and Simon, 1990, p.147). But the isomorphism can be perceived only when the change of state viewpoint is adopted. Actually this interpretation allows understanding the action as removing something from one place and putting into another for move problems and as withdrawing a given size and then giving a new one in the case of change problems.

We claim that the context (the nature of the change) is what induces a particular coding of the operator that is more or less close to what is required for constructing the adequate operator and optimizing problem solution.

Within each of these two classes, the version which emphasizes the change-ofstate viewpoint is compared with the version which emphasizes the transformation viewpoint. The predicted difference in difficulty should be manifested in solution time, number of moves, and violations of the rules in the instructions. Moreover, in the problems in which the transformation viewpoint is made more salient, we should observe misinterpretations which can be found only if the transformation viewpoint is adopted, namely, the idea that it is not possible to go directly from one extreme state to the other.

In our second experiment, we added further controls intended to determine whether the spatial memory load hypothesis can be excluded as a possible cause for the observed differences.

\section{EXPERIMENT 1}




\section{Method}

Subjects. The subjects were 60 college students attending the University of Paris 8 .

Problems. The four problems were Move-Change of state (The Tower of Hanoi), MoveTransformation (The Lift), Change-Change of state (The Counters), ChangeTransformation (The Change of Size). We attempted to make the presentation conditions as comparable as possible in the move and change problems. The objects were presented at three different places, with several objects in the same place in some cases of the move problems, and only one object per place for the change problems. The four problems are depicted in Figure 1, as they appeared on the screen of a Macintosh computer.

(Figure 1 about here)

As can be seen in Figure 1a, in the Tower of Hanoi version three blocks of different sizes (small, medium, large) are in three different places (left, middle, right). On the top, the initial state is shown and in the box at the bottom the state to be reached is shown. Moves are made by clicking first on the block to be moved and then on the place to be reached. In the Lift problem (Figure 1b), three persons of different sizes (short, medium, tall) were placed on different floors (ground floor, middle floor, upper floor), such that the changeable value was the place and the fixed value was the size of the person, as in the Tower of Hanoi problem, where place is variable and size, constant. The initial state appears on the right and the final state on the left. Moves were made by clicking first on the person to be moved and then on the desired floor.

In the Counter problem (Figure 1c), three counters were placed on the left, in the middle, and on the right, respectively. The lower part of the figure shows the initial 
state, and the upper part, the goal. The figure was changed by clicking on the desired number (in small characters on the left and right) of the counter to be changed.

In the Change of size problem three blocks of different sizes (small, medium, large) were placed in three different places (left, middle, right) each held by a person (figure 1d). The final state to reach was shown at the bottom of the screen. Changes were made by clicking on the block to be changed and then on the desired size (in the upper frame). In this version, as in the counter version, size was changeable and place was constant. This problem was expected to be easier than the monster problem, since the constant was position instead of size, and, more importantly, the changes and states were made visible.

The rules for each problem are presented in the figures showing the material. All of the problems required five steps to be solved in the optimal way. Referring to Figure 1(a,b,c,d), the initial state may be coded 312 and the final state 123, with the first figure being the value of the changeable attribute of object 1 (small disk or left square), the second figure being the value of the changeable attribute of object 2 (medium disk or middle square) and the third figure being the value of the changeable attribute of object 3 (large disk or right square). The values of the changeable attribute are:

\begin{tabular}{|l|l|l|l|l}
\hline & Tower of Hanoi & Lift & Counters & Blocks \\
\hline 1 & left & ground floor & 1 & small \\
\hline 2 & middle & middle floor & 2 & medium \\
\hline 3 & right & upper floor & 3 & large \\
\hline
\end{tabular}

Design. The subjects were evenly assigned to each of the four conditions, making 15 subjects per condition. 
Procedure. The subjects were tested individually. The problems were presented on the screen of a Macintosh micro-computer. Subjects were asked to solve the problem on the screen and were given the following instructions: "Solve the following problem by changing the initial state into the final one. The change is subject to the rules shown on the right. When you press on the mouse, the rules will disappear, but you can make them appear at any time by clicking on the right part of the screen. If you request an illegal change, it will not occur and the rule which prohibits the change will appear at the bottom of the screen. You may take as much time as you need, but remember that the computer records the time spent. You may start whenever you are ready." Subjects were shown how to execute a move or a change using the mouse. The moves made by the subjects and the time elapsed since the last response were recorded automatically by the computer.

\section{Results}

The results will be presented in two parts, one per hypothesis tested: (1) the effect of the point of view and of the type of operator on performance, and (2) the effect of these two factors on the understanding of the instructions.

1. Point-of-view and type-of-operator effects on performance. Two dependent measures of performance were considered: number of moves and solution time.

Number of moves. The data for the number of moves are presented in Figure 2. It is obvious that problems in which the change-of-state point of view was emphasized required a smaller number of moves to be solved. The point-of-view effect was significant $[\mathrm{F}(1,56)=7.48 ; \mathrm{p}=.008]$. Using Bayes-fiducial methods (see Appendix for a description), we may conclude that with a credibility level of .90 , the true value of the difference is larger than 7.8. As this value is half the number of moves observed in situations with emphasis on the change of state, we may conclude that the difference is 
large. At the descriptive level, change problems seem to take more moves to solve than move problems $\left(\mathrm{d}_{\mathrm{obs}}=5.5\right)$, but the difference was not significant $[\mathrm{F}(1,56)=1.037$; $\mathrm{p}=.31]$. However, it cannot be considered negligible $\left[\mathrm{p}\left(\delta_{-}<-12.69 \_\right)=.90\right]$ : one may conclude with a credibility level of .90 that the absolute value of the difference was smaller than 12.69 , a value which certainly cannot be regarded as negligible. The same conclusions can be drawn for the interaction between the two factors: the effect was not significant $[\mathrm{F}(1,56)=2.505 ; \mathrm{p}=.11]$ but cannot be said to be negligible $\left[\mathrm{p}\left(\delta_{-}<\_15.72 \_\right)=.90\right]$.

(Figure 2 about here)

These results are consistent with the hypothesis that the point-of-view effect would be strong. The effect of the type of the operator was not significant, but cannot be discarded.

Solution time. The data on solution time are presented in Figure 3. The average solution time for the versions which stressed the transformation was twice as long as the average solution time for the versions stressing the change of state. The analysis of variance yielded a significant point-of-view effect $[F(1,56)=12.097 ; p=.001]$. Bayesfiducial methods showed that $\mathrm{p}(\mathrm{d}>3.50)=.90$ and $\mathrm{p}(\mathrm{d}>2.83)=.95$. As these values are more than half of the observed solution time in the situations where the change-of-state point of view was more salient, we may conclude that the difference was great. The effect of the problem type factor (move vs. change) was not significant $[\mathrm{F}(1,56)=2.35$; $\mathrm{p}=.13]$, but it cannot be called negligible $\left[\mathrm{p}\left(\delta_{-}<{ }_{-} 5.12\right]=.90\right]$. The interaction between the two factors was significant $[\mathrm{F}(1,56)=7.193 ; \mathrm{p}=.009]$ and can be considered relatively large $\left(\mathrm{d}_{\mathrm{Obs}}=4.55\right.$ and $\left.\mathrm{p}(\mathrm{d}>2.20)=.90\right)$. Thus, the effect of the point-of-view factor was greater in the change problems than in the move problems and was 
significant in the change problems only. So, while the effect on solution time reported by Kotovsky, Hayes, and Simon (1985) may be partly explained by a point-of-view effect, the nature of the operator also plays a role, since the point-of-view effect was weaker in move problems. For performance as a whole, the point-of-view effect was greater in the case of size changes than in the case of place changes. This point will be discussed later.

(Figure 3 about here)

\section{Point-of-view and type-of-problem effects on the understanding of the instructions}

We predicted that if the problem context focused the subject's attention on the process rather than on the change of state, and thus making it more difficult to break the action down into subgoals, the problem would be harder to solve because rules 2 and 3 could not be immediately interpreted as prerequisites for getting out of one state and getting into a new one, respectively. A second prediction is that we should only observe misinterpretations prohibiting a direct change from one extreme state to another in the problems where the transformation process is made more salient (The lift and the Change of Size), since with such an interpretation, a transition between extreme states is thought to require going through the intermediate state. We shall now consider the data relevant to testing these two predictions.

Number of violations of rule 2. This rule states the condition for taking an object from its place in the move problems, and for removing the size (or the value) of an object in the change problems. The average number of violations of this rule for each problem is shown in Figure 4. As can be seen, violations were less frequent when the change-ofstate viewpoint (rather than the transformation) was made more salient. This difference was significant $[\mathrm{F}(1,56)=4.58 ; \mathrm{p}=.03]$ and may be regarded as relatively large 
$\left(d_{o b s}=3.55\right.$ and $\left.p(d>1.39)=.90\right)$. The effect of the type of operator was also significant $[\mathrm{F}(1,56)=5.58 ; \mathrm{p}=.02]$ and the difference can be considered relatively important $\left(\mathrm{d}_{\mathrm{obs}}=3.25\right.$ and $\left.\mathrm{p}(\mathrm{d}>1.04)=.90\right)$. The interaction between the two factors was not significant $[\mathrm{F}(1,56)=2.05 ; \mathrm{p}=.15]$ but the effect cannot be regarded as negligible $\left[\mathrm{p}\left(\delta_{-}<-4.53 \_\right)=.90\right]$.

(Figure 4 about here)

Number of violations of rule 3. This rule states the condition for giving an object a new place (move problems) or a new size or value (change problems). As can be seen in Figure 5, there were more violations of this rule in the problems where the attention was focused on transformation. This difference was significant $[F(1,56)=9.93 ; p=.002]$ and may be considered large $\left[\mathrm{d}_{\mathrm{obs}}=5.85, \mathrm{p}(\mathrm{d}>3.19)=.90\right)$, and $\left.\mathrm{p}(\mathrm{d}>2.76)=.95\right]$. The effect of the type of operator was not significant $[F(1,56)=2.048 ; p=.15]$, but cannot be considered negligible $\left[\mathrm{p}\left(\delta_{-}<-7.04\right)=.90\right]$. The interaction was not significant $[\mathrm{F}(1,56)=.89]$, but the effect cannot be regarded as negligible $\left[\mathrm{p}\left(\delta_{-}<{ }_{-} 4.16 \_\right)=.90\right]$.

(Figure 5 about here)

Misinterpretation of allowed transitions between states. If our interpretation is correct, we should observe specific rule misinterpretation in both of the problems (figures $1 \mathrm{~b}$, 1d) which emphasize transformation, namely, that subjects would believe it was prohibited to shift from one extreme state to the other (from left to right or small to large) without passing through an intermediary state. This misinterpretation can be considered as an additional constraint on the moves (in addition to the constraints in the instructions).

Richard, Poitrenaud, and Tijus (1993) have shown that the rules in the instructions, as well as heuristics and goals, can be represented as constraints, where a 
constraint is defined as a restriction on the set of possible moves. For any state in the problem space, a constraint allows a given set of moves and prohibits others. This may be expressed as a matrix where the rows stand for moves and the columns stand for states. Each cell in this matrix contains a Boolean value of either 0, meaning that the move is allowed by the constraint, or 1 , meaning that the move is prohibited by the constraint. Thus, the matrix filters the moves for each state, allowing only those which fit the constraint. Table 1 gives the matrix representing the constraint that prohibits transposition between extreme states, which, according to our hypothesis, is a misenterpretation induced by the transformation point of view.

(Table 1 about here)

In state 111 (first column), which for the Tower of Hanoi problem means that the three disks are on the left, the misinterpretation represented by this constraint prohibits moves 13, 23, and 33, which directly transfer the small, medium, and large disk, respectively, from the left location to the right location. This is expressed as a 1 in the cells in the first column which correspond to these moves. According to this misinterpretation, in state 111 of the change problem (small left block, small middle block, and small right block), directly changing a block from the small size to the large one is not allowed. Note that this matrix does not show the moves prohibited by rules 2 and 3 in the instructions: these rules may be represented by similar tables (see Richard, Poitrenaud, and Tijus, 1993).

In order to be able to determine whether the protocols were consistent with this constraint, an index of the relevancy of the constraint to the protocol was computed. The index was defined as follows and was computed for each trial.

(i) if the move is consistent with the constraint (there is a 0 in the cell corresponding the state and the move): 


$$
\mathrm{r}=1-\quad \frac{\text { number of moves allowed by the constraint }}{\text { number of possible moves }}
$$

The number of moves allowed by the constraint are shown in the matrix row labelled "consistent" and the number of possible moves are shown in the last row.

(ii) if the move is not consistent with the constraint (the corresponding cell contains a 1$)$ :

$$
r=-\quad \frac{\text { number of moves allowed by the constraint }}{\text { number of possible moves }}
$$

The mean value of the index across the trials of each subject's protocol was computed. The ratio mean number of moves allowed by the constraint/number of possible moves gives the probability that a random choice will generate a move that is consistent with the constraint. So the relevancy index mean would be equal to 0 in the case of a random choice: it is the expected value if the constraint is not relevant to the protocol. A positive value indicates that the choices tended be consistent with the constraint.

The mean relevancy value of this constraint per trial was computed for each subject in each situation. The means of these values over subjects for each condition are shown in Figure 6. A comparison of these values shows to what extent subjects in each problem conformed to this constraint.

(Figure 6 about here)

As can be seen, the mean relevancy index of this constraint was higher in the two versions which emphasized the transformation, while its value was close to 0 in the two versions in which the result was emphasized. The point-of-view effect was significant $\left[\mathrm{d}_{\mathrm{obs}}=0.055\right.$ and $\left.\mathrm{F}(1,56)=3.826 ; \mathrm{p}=.05\right]$, and pooling the move and change groups in each point-of-view condition, it can be shown that $\mathrm{p}(\mathrm{d}>0.02)=.90$. The type-of-problem effect was not significant $[F(1,56)=.005 ; \mathrm{p}<05]$, but the effect cannot be considered negligible $\left[\mathrm{p}\left(\delta_{-}<\_.048 \_\right)=.90\right]$. In the same way, the interaction between the two 
factors was not significant $[\mathrm{F}(1,56)=.13 ; \mathrm{p}=<.05]$, but the effect cannot be said to be negligible $\left[\mathrm{p}\left(\_\_<. .050 \_\right)=.90\right]$.

In order to grasp what a mean relevancy index of 0.055 means, remember that it is a per-trial mean. If we consider Table 1, assuming that each state has been visited an equal number of times, it can be shown that a protocol exhibiting this constraint on every trial would have a mean index of 0.33 . As the moves required in the final path [312 -> 11; 112 -> 33; 113 -> 13; 313 -> 22; 323 -> 31; 123] show three violations of this constraint versus two which abide by it, the mean relevancy of the constraint for the final path is $(-0.055)$. So a mean value of 0.055 for a protocol of 15 trials implies that the mean relevancy value of the constraint for the first 10 trials was 0.110 . In other words, this means that the constraint is present in a third of the trials for each subject, or that the constraint is present in the whole protocol for a third of the subjects. Thus, considering the fact that the transformation point of view makes this misconception possible but not necessary, it appears that in situations assumed to induce a transformation point of view, a substantial proportion of the subjects adopted this misinterpretation.

\section{Discussion}

These results are consistent with the hypothesis that the point of view suggested by the problem context is an important factor in determining its difficulty. A problem is difficult when the context draws the subject's attention towards the way the transformation is produced rather than towards the initial and final states. It is more difficult to adopt the adequate point of view in change-of-size problems because in problems of this kind, the action of changing a size is interpreted as a continuous process: the interpretation of the action centers on the transformation because an analogy with growth is drawn. Thus, if the change-of-state point of view is emphasized in these problems, they become less difficult to solve. 
However, the effect of the type of operator cannot be discarded, and the most radical version of our hypothesis that point of view is the only factor determining difficulty was not supported by the results. In every analysis there was some indication of an effect of the type of operator (move versus change) and of an interaction effect: these effects were not significant in most analyses, but were so in some, and in all cases, the effect could not be regarded as negligible. Moreover, it appears that the change-of-size condition was responsible for the major part of the difference, in agreement with previous results. Actually this condition was the only one in which the alternative values of the changeable attribute were not physically present. In the move problems, the possible places were present, and in the counter problem, the other possible values for a counter appeared on the left and right (see Figure 1). So the spatial memory load hypothesis proposed by Kotovsky, Hayes, and Simon (1985) may account for these results.

Experiment 2 was conducted as a complement to experiment 1. It was devised in order to test whether the type-of-operator effect can be explained by the memory load hypothesis. There are two ways of testing this hypothesis. The first is to use a change-of-size problem and compare a condition where the alternative sizes for an object are not visible (as in the condition studied) with a condition where they are. This was the aim of experiment $2 \mathrm{a}$. The second way is to use the counter problem and compare the condition where the alternative values of each counter are visible (already studied) with a condition where they are not. This was the purpose of experiment $2 \mathrm{~b}$. The memory load hypothesis predicted a difference in each case. 


\section{EXPERIMENT 2}

\section{Experiment 2a}

\section{Method}

Subjects. Thirty new subjects were tested in this experiment. They were all students at the University of Paris 8 .

Problems. For the condition where the alternative sizes of the cubes were not visible (see Figure 1d of experiment 1), the same material was used. For the condition where the alternative sizes were visible, a condition similar to the dish-change problem studied by Kotovsky, Hayes, and Simon (1985) was used. On the top of the computer screen above each cube, the two sizes other than the current one were displayed (see Figure 7). To make the action meaningful, the size change was presented as an exchange, as in Kotovsky, Hayes, and Simon (1985). In order to change a size, the subject had to click on the cube to be changed and then on the cube of the size desired, exactly as in the first condition where the alternative sizes for each cube were not visible.

(Figure 7 about here)

Design. Fifteen subjects were tested on the change of size problem with visible alternative values, and fifteen subjects solved the problem in the condition with invisible alternative values.

Procedure. Subjects were tested individually under the same conditions as in experiment 1 . They were given the same instructions and were shown how to make a move using the mouse.

Results The results are presented in Table 2 for the various dependent variables (number of moves, time to solution, violations of rule 2, and violations of rule 3 ). 
(table 2 about here)

The values obtained for the condition with invisible alternative values were quite close to those obtained with visible alternative values. One can see that the differences between the conditions with visible vs. invisible alternative sizes were small, and that for three out of four dependent variables, performance was better in the condition where the alternative sizes were not visible. In all cases the differences were far from significant, but cannot be said to be negligeable. Kotovsky, Hayes, and Simon (1985) found that their "dish-change" problem was somewhat easier than the monster problem. This discrepancy between our results and theirs may be explained by the fact that their subjects had to physically execute the action of exchanging balls of different sizes with those in the reserve dish in order to change the size of the ball in the dish, while in our problem subjects did exactly the same as in the change problem, with the notion of exchange only being present in the instructions. Probably, performing the objectexchanging action is a stronger cue for activating the meaning of "exchange" and leading to the interpretation that an exchange is a kind of double move. In fact, this difference in the results supports the point-of-view hypothesis: the physical context of the exchange used by Kotovsky, Hayes, and Simon emphasizes the change-of-state viewpoint (to exchange a disk, one had to "take it", then take another dish, and then "put" the latter in the vacant place). This difference in context may account for the difference in results.

\section{Experiment 2b}

\section{Method}


Subjects. Thirty new subjects were tested in this experiment.

Problems. The condition where the alternative values of the counters were visible was the condition used in experiment 1 (see Figure 1c). For the condition where the alternative sizes were not visible, the same device was used, except that the values other than the current one were not shown on the left and right of the counter (see Figure 8).

(Figure 8 about here)

Design. Fifteen subjects were tested on the counter problem with visible alternative values, and fifteen subjects solved the problem in the condition with invisible alternative values.

Procedure. Subjects were tested individually under the same conditions as in experiment 1 . They were shown how to change the values of a counter by clicking on the left and clicking on the right.

\section{Results}

The results are presented in Table 3 for the various dependent variables. The values obtained for the condition with visible alternative values were very close to those obtained for the same condition in experiment 1 . One can see that performance was always slightly better in the condition where the alternative values of counters were visible, but the differences were not significant. The differences cannot however be said to be negligeable, except perharps in the case of the number of violations of rule 2 , if we admit that 1.20 is a value which is negligible.

(Table 3 about here) 


\section{Discussion}

Considering the results of experiments $2 \mathrm{a}$ and $2 \mathrm{~b}$, we can not conclude that the memory load hypothesis is responsible for the type-of-operator effect observed in experiment 1 . The differences were not significant: the fact that the differences support this hypothesis in one case and refute it in the other suggests that another factor must be found to explain the difficulty of the change-of-size problem.

\section{GENERAL DISCUSSION}

The results of experiments 1 and 2 support the predictions of the point-of-view hypothesis and show that the type of operator also has an effect, and that this is in fact an interaction effect: adopting a different point of view was more difficult in the change-of-size problem than in the lift problem, where the operator was a place change. Although these results showed that this effect took place during the building of the problem representation, they did not precisely indicate its locus.

In summary of the data obtained through the two experiments, we may conclude the following:

(i) The difference in point of view suggested by the "embodiment" or cover story of the problem is an important factor in determining the difficulty of a problem, measured as the number of moves, the time needed to solve the problem, and the understanding of the instructions.

(ii) As predicted, the misinterpretation that only nearby changes are allowed was only observed for problems where the context induced the transformation point of view.

(iii) The potential effect of the type of operator cannot be excluded, since performance was always slightly (and sometimes significantly) poorer when the operator was a size change. At the same time, the difference due to the point of view was always slightly larger (sometimes significantly) for changes than for moves. However, it appears that 
this effect cannot be explained by the spatial memory load hypothesis formulated by Kotovsky, Hayes, and Simon (1985), since reducing the memory load by making the alternative values of the changeable attribute visible did not produce significant and stable differences.

Our results stress the importance of the construction of the problem space. The graph usually presented for the Tower of Hanoi problem and its isomorphs is the graph of an expert who has constructed the prerequisite of the operator: a disk may be moved to a place if (i) there is no smaller disk on top of it and (ii) there is no smaller disk at that place. The transitions between states in this graph represent the transformations allowed by the operator. But before arriving at this terminal problem space, it is necessary to build a representation whose graph is much more complex. This graph has two suboperators (leaving a state, entering a state) and contains a much larger number of nodes and transitions. Moreover, it must account for all states defined by the fact that one disk is being held in the subject's hand (two disks being on the board), and all possible transitions between all these states. This graph is built when rules 2 and 3 in the instructions are understood as prerequisites for leaving a state and entering a new one, respectively. It is impossible to directly construct the expert graph based on the operator alone. Before a global operator may be put to use as described by the meansend heuristic (Newell and Simon, 1972), a graph based on the suboperators must be devised, the prerequisite for each suboperator identified, and all of the later integrated in order to obtain the prerequisite for the operator.

From this perspective, the first and crucial step in problem solving is a semantic analysis of the situation which involves two aspects: selecting the appropriate point of view about the action described in the instructions and transforming the rules stated in the instructions into prerequisites for the operator. This analysis suggests that planning cannot take place until an appropriate understanding of the problem has been gained. It explains why adults, who exhibit sophisticated planning in Tower of Hanoi problems, 
solve difficult isomorphs using the apparent trial-and-error tactics characteristic of children solving Tower of Hanoi problems.

There are many other problems which are rendered difficult by a context which suggests an immediate interpretation in terms of transformation when the solution requires a change-of-state point of view. A good example is the following jar problem (Leonard, 1984). There are two jars, one is full of wine and the other is full of water. Someone takes a glass of wine from the first and pours it into the second; then a glass of the mixture is taken from the second jar and poured into the first. The question is: "Is there more wine in the water or more water in the wine?" This problem appears to be quite difficult because subjects try to determine what happens at each step of the transformation: they compute sophisticated proportions and are usually mistaken. The solution comes to light when one considers the result of the two transformations. Each jar contains the same quantity of liquid before and after, since the same quantity of liquid has been exchanged, i.e. the wine taken from the first jar is necessarily in the second and therefore ought to be exactly balanced by the water. Otherwise there would not be the same quantity as before. Consequently, there must be as much wine in the water as water in the wine.

The selection of the appropriate point of view for solving the problem does not only concern Tower of Hanoi isomorphs. It is well known that solving very difficult problems requires a change in point of view (Ohlsson, 1992). Among such problems are the well-known monk problem, the cyclists and the fly problem, and the mutilated checkerboard problem (Wickelgren, 1972; Kaplan and Simon, 1990).

These problems are related to the "insight" problems of the Gestalt tradition, to which recent attention has been attracted by Ohlsson (1984a, 1984b) and Keane (1989). The only difference is that insight is regarded as sudden, but as Ohlsson (1992) argues, this aspect is controversial and is not a major issue. Paradoxically, insight problems have not been studied extensively, except for the nine dots problem (Weisberg and 
Alba, 1982). The reason for this is probably that the exploration is almost entirely covert and unconscious, making a precise analysis of the process of insight impossible.

Several attempts have been made to give an information processing explanation of "impasse" and insight phenomena. (Simon, 1986; Ohlsson, 1992). Dealing with the same question, we proposed a constraint model of problem solving in which problem solving restructuration is seen as elimination of implicit constraints. (Richard, Poitrenaud, Tijus, 1993; Clément, 1994). An impasse occurs when, given the present set of constraints, no response is allowed. In such a situation a constraint has to be relaxed: it is assumed that the constraints are ordered (according to importance), and that the least important one is temporally removed in order to allow an action. A constraint which is temporally relaxed is not definitively eliminated, so that restructuration may be progressive as Ohlsson argues (1992).

The present paper attempted to explain how the construction of the operator which takes place during the exploratory phase depends on the context of the situation, and above all, on how relevant the semantic aspects of the problem are to the interpretation of the situation. 


\section{References}

Bernicot, J. (1981). Le développement des systèmes sémantiques des verbes d'action. Monographies Françaises de Psychologie. Paris: Editions du CNRS

Clément, E. (1990). La représentation de l'action: l'interprétation des consignes de problèmes isomorphes. Mémoire de DEA. Université de Paris 8.

Clément, E. (1994). La représentation de l'action: l'interprétation des consignes dans des problèmes isomorphes. Thèse de Doctorat. Université de Paris 8. Janvier 1994.

Clément, E. et Richard, J.F. (1992). Compréhension des énoncés de problèmes et construction des opérateurs. International Journal of Psychology. 27, 3/4. 164.

Clément, E. et Richard, J.F. (1992). The understanding of the instruction in isomorph problem. Abstracts Fith Conference. Congrès de ESCP, Paris, France, Septembre 12-16, 1992, p 90-91.

Gick, M. L., \& Holyoak, K. J. (1980). Analogical problem solving. Cognitive Psychology. 12. 306-355.

Gick, M. L., \& Holyoak, K. J. (1983). Schema induction and analogical transfer. Cognitive Psychology. 15. 1-38.

Hayes, J. R., \& Simon, H. A. (1977). Psychological differences among problem isomorphs. In N.J. Castellan, D.B. Pisoni, \& G.R. Potts; (Eds). Cognitive theory. Hillsdale, NJ: Erlbaum.

Kaplan, C.A. \& Simon, H.A. (1990). In search of insight. Cognitive Psychology, 22, 374-419

Keane, M. (1989). Modelling problem solving in Gestalt 'insight' problems. The Irish Journal of Psychology, 10, 201-215

Kotovsky K, Hayes J.R, Simon H.A.(1985). Why are some problems hard? Evidence from Tower of Hanoi. Cognitive Psychology, 17. 248-294.

Kotovsky, K. \& Fallside D.(1989). Representation and transfer in problem solving. In: Kotovsky, K. (Ed.) Complex information processing. What has Simon brought? 21st symposium of the Carnegie Mellon Institute. LEA

Kotovsky, K. \& Simon, H.A. (1990). What makes some problems really hard: explorations in the problem space difficulty. Cognitive Psychology, 22, 143183.

Léonard, F. (1984). L'interprétation des effets de contenu: changement de réponse ou changement de raisonnement. Cahiers de Psychologie Cognitive, 4, 475-493.

Newell, A \& Simon, H.A. (1972). Human Problem Solving. Englewood Cliffs, NJ: Prentice Hall.

Ohlsson, S. (1984a). Restructuring revisited. I. Summary and critique of the Gestalt theory of problem solving. Scandinavian Journal of Psychology, 25, 65-78.

Ohlsson, S. (1984b). Restructuring revisited. II. An information processing theory of restructuring and insight. Scandinavian Journal of Psychology, 25, 117-129

Ohlsson (1992). Information-processing explanations of insight and related phenomena. In M. Keane \& K. Gilhooly (Eds.) Advances in the psychology of thinking, Vol. 1. London: Harvester Wheatsheaf.

Reed, S. K., Ernst, G. W., \& Banerji, R. (1974). The role of analogy in transfer between similar problem states. Cognitive Psychology. 6. 436-450. 
Richard, J.F. \& Poitrenaud, S. (1988). Problématique de l'analyse des protocoles individuels d'observations comportementales. In J.P Caverni, C. Bastien, P. Mendelsohn, \& G. Tiberghien. (Eds). Psychologie cognitive: concepts et méthodes.(405-426). Grenoble. Presses Universitaires de Grenoble.

Richard, J.F. (1990). Les Activités Mentales. Comprendre, raisonner, trouver des solutions. Armand Collin. Paris.

Richard, J.F., Poitrenaud, S., \& Tijus, C. (1993). Problem solving restructuration: Elimination of implicit constraints. Cognitive Science, 17,497-529.

Rouanet, H. (in press). Bayesian inference for assessing importance of effects. Psychological Bulletin.

Rouanet, H., Lépine \& D. Holender, D. (1978). Model acceptability and the use of Bayes-fiducial procedures for validating models. In J. Requin (Ed.) Attention and performance VII. Hillsdale, NJ: Erlbaum 687-701..

Rouanet, H., Lépine, D \& Pelnard-Considéré.(1976). Bayes-fiducial procedures as practical substitutes for misplaced significance testing: An application to educational data. In D.N.M. de Gruijter and L.J. Th. Van den Kamp (Eds.) Advances in Psychological and Educational Measurement. New York: Wiley 33-50.

Simon, H. A. (1986). The information processing explanation of Gestalt phenomena. Computers in Human Behavior. 2. 241-255.

Simon, H. A., \& Hayes, J. R. (1976). The understanding process: problems isomorphs. Cognitive Psychology. 8.165-190.

Weisberg, R. \& Alba, J.W. (1982). Problem solving is not like perception: more on Gestalt-theory. Journal of Experimental Psychology General, 111(3), 326-330.

Wickelgren, W. (1972). How to solve problems: Elements of a theory of problem solving. San Francisco: Freeman and Co 


\title{
E. Clément and J.F. Richard
}

\author{
APPENDIX \\ Bayes-fiducial methods to assert the importance or the negligibility of an effect \\ (from Rouanet, in press)
}

Let $d$ be the observed effect (difference between the means of two independent samples), $\delta$ the effect in the population and $s$ the unbiased estimate of the standard mean deviation of the two samples. The significance test approach consists of computing the sampling distribution of the $t$ statistic under the null hypothesis that $\delta=0$ and positioning the observed value of $t\left(t_{\mathrm{obs}}\right)$ on this distribution. In the case of independent samples, $t=-\frac{d}{s \sqrt{1 / n^{\prime}+1 / n^{\prime \prime}}}, n^{\prime}$ and $n^{\prime \prime}$ being the number of observations in each sample. If we let $e=s \sqrt{1 / n^{\prime}+1 / n^{\prime \prime}}, t=d / e$. If the probability that $t$ exceeds $t_{\mathrm{obs}}$ is small enough (say $.05)$, the null hypothesis is rejected.

The Bayesian approach consists of making a probabilistic statement about $\delta$ (the unknown parameter), given the data. Assuming a no-informative prior (the prior distribution of $\mathrm{d}$ is uniform), it can be shown (see Rouanet, Lépine \& Pelnard-Considéré, 1976; Rouanet, Lépine \& Hollender, 1978; Rouanet, in press) that, given $d=d_{\text {obs }}$ (observed value of $d$ ), the posterior distribution of $\delta$ is such that ( $\delta$ $d_{\mathrm{obs}}$ ) $/ e$ is distributed as an elementary $t_{q}$ variable ( $q$ being the number of degrees of freedom). The mean of the probability distribution of $\delta$ is $d_{\mathrm{obs}}$ and its standard deviation is $e$.

Using this distribution, it is possible to test the importance of an effect as well as the negligibility of an effect. In order to test the importance of an effect, we compute the value $d_{c}$ of $\delta$ (using a $t$ table) such that $\mathrm{P}\left(\delta>d_{c}\right)=.90$ : if the value $d_{c}$ may be considered as high (according to our knowledge of the domain), then with a credibility level of .90 we may conclude that the difference is great It is possible to choose a higher value for the credibility level, but choosing .90 seems reasonable. The usual significance level may be reinterpreted in the following way in the Bayesian framework: if the null hypothesis is rejected at probability level of $p$ (one-sided test), then $\mathrm{P}(\delta>0)=1-p$.

In order to test the negligibility of an effect, we compute the size of the interval_ $d_{-}$around 0 such that $\mathrm{p}\left(\delta_{-}<_{-} d_{-}=.90\right.$. If this interval is small enough as to be considered as negligible, then with a credibility level of .90 we may conclude that the difference is negligible. Testing the importance of an effect goes beyond asserting the significance of an effect, since the conclusion that the effect is significant means only that it is greater than zero: it does not tell how large it is. Testing for the negligibility of an effect is appropriate when we want to show that there is no effect, in which case a conclusion of no-significance is not adequate. A conclusion of negligibility is actually very difficult to obtain, because it requires both a small observed effect and a low value of $e$ (which requires large samples). 
Table 1.

Matrix of the constraint related to the transformation point of view: "it is prohibited to jump from one extreme state to the other". The states are in columns, and are coded, as said previously, with the value of the variable attribute for each object. The actions are shown in rows: 13 means giving value 3 to object $1 ; 32$ means giving value 2 to object 3 , etc. The three rows at the bottom indicate the number of actions allowed by the constraint in each state, the number of actions prohibited, and the total number of possible actions, respectively. Null actions such as 11,21 or 31 in state 111 are not considered: see the blank cells (for example taking a disk and laying it down again in the same place).

\begin{tabular}{|c|c|c|c|c|c|c|c|c|c|c|c|c|c|c|c|c|c|c|c|c|c|c|c|c|c|c|c|}
\hline object 1 & 1 & 3 & 3 & 2 & 2 & 1 & 1 & 3 & 2 & 2 & 2 & 3 & 3 & 1 & 1 & 2 & 3 & 3 & 1 & 1 & 2 & 2 & 1 & 1 & 2 & 3 & 3 \\
\hline object 2 & 1 & 1 & 2 & 2 & 2 & 2 & 3 & 1 & 1 & 1 & 3 & 3 & 3 & 3 & 2 & 2 & 2 & 1 & 1 & 1 & 1 & 3 & 3 & 2 & 3 & 2 & 3 \\
\hline object 3 & 1 & 1 & 1 & 1 & 3 & 3 & 3 & 3 & 2 & 1 & 1 & 1 & 2 & 2 & 2 & 2 & 2 & 2 & 2 & 3 & 3 & 3 & 1 & 1 & 2 & 3 & 3 \\
\hline \multicolumn{28}{|l|}{ Move } \\
\hline 13 & 1 & & & 0 & 0 & 1 & 1 & 0 & 0 & 0 & 0 & & & 1 & 1 & 0 & & & 1 & 1 & 0 & 0 & 1 & 1 & 0 & & \\
\hline 12 & 0 & 0 & 0 & & & 0 & 0 & 0 & & & & 0 & 0 & 0 & 0 & & 0 & 0 & 0 & 0 & & & 0 & 0 & & 0 & 0 \\
\hline 11 & & 1 & 1 & 0 & 0 & & & 1 & 0 & 0 & 0 & 1 & 1 & & & 0 & 1 & 1 & & & 0 & 0 & & & 0 & 1 & 1 \\
\hline 23 & 1 & 1 & 0 & 0 & 0 & 0 & & 1 & 1 & 1 & & & & & 0 & 0 & 0 & 1 & 1 & 1 & 1 & & & 0 & & 0 & \\
\hline 22 & 0 & 0 & & & & & 0 & 0 & 0 & 0 & 0 & 0 & 0 & 0 & & & & 0 & 0 & 0 & 0 & 0 & 0 & & 0 & & 0 \\
\hline 21 & & & 0 & 0 & 0 & 0 & 1 & & & & 1 & 1 & 1 & 1 & 0 & 0 & 0 & & & & & 1 & 1 & 0 & 1 & 0 & 1 \\
\hline 33 & 1 & 1 & 1 & 1 & & & & & 0 & 1 & 1 & 1 & 0 & 0 & 0 & 0 & 0 & 0 & 0 & & & & 1 & 1 & 0 & & \\
\hline 32 & 0 & 0 & 0 & 0 & 0 & 0 & 0 & 0 & & 0 & 0 & 0 & & & & & & & & 0 & 0 & 0 & 0 & 0 & & 0 & 0 \\
\hline 31 & & & & & 1 & 1 & 1 & 1 & 0 & & & & 0 & 0 & 0 & 0 & 0 & 0 & 0 & 1 & 1 & 1 & & & 0 & 1 & 1 \\
\hline consistent & 3 & 3 & 4 & 5 & 5 & 4 & 3 & 3 & 5 & 4 & 4 & 3 & 4 & 4 & 5 & 6 & 5 & 4 & 4 & 3 & 4 & 4 & 3 & 4 & 5 & 4 & 3 \\
\hline inconsistant & 3 & 3 & 2 & 1 & 1 & 2 & 3 & 3 & 1 & 2 & 2 & 3 & 2 & 2 & 1 & 0 & 1 & 2 & 2 & 3 & 2 & 2 & 3 & 2 & 1 & 2 & 3 \\
\hline total & 6 & 6 & 6 & 6 & 6 & 6 & 6 & 6 & 6 & 6 & 6 & 6 & 6 & 6 & 6 & 6 & 6 & 6 & 6 & 6 & 6 & 6 & 6 & 6 & 6 & 6 & 6 \\
\hline
\end{tabular}


Table 2.

Results of experiment 2a: change of size (alternative values visible and alternative values not visible).

\begin{tabular}{|c|c|c|c|c|c|}
\hline $\begin{array}{l}\text { dependent } \\
\text { variable }\end{array}$ & condition & subjects & mean & $\begin{array}{l}\text { Memory load } \\
\text { hypothesis }\end{array}$ & $\begin{array}{l}\text { Bayesian } \\
\text { test }\end{array}$ \\
\hline \multirow{3}{*}{$\begin{array}{l}\text { number of } \\
\text { moves }\end{array}$} & alternative & & & \multirow{3}{*}{$\begin{array}{l}F(1,28)=0.05 \\
p=.81\end{array}$} & \multirow{3}{*}{$\begin{array}{l}\mathrm{p}\left(\delta_{-} \_-57.28 \_\right) \\
=.90\end{array}$} \\
\hline & visible & 15 & 39.93 & & \\
\hline & alternative values not & & & & \\
\hline \multirow[t]{3}{*}{ solution time } & alternative & & & \multirow{3}{*}{$\begin{array}{l}F(1,28)=.65 \\
p=.42\end{array}$} & \multirow{3}{*}{$\begin{array}{l}\mathrm{p}\left(\delta_{-} \_<8.67 \_\right) \\
=.90\end{array}$} \\
\hline & visible & 15 & 14.54 & & \\
\hline & $\begin{array}{l}\text { alternative values not } \\
\text { visible }\end{array}$ & 15 & 11.72 & & \\
\hline \multirow{3}{*}{$\begin{array}{l}\text { violations } \\
\text { of rule } 2\end{array}$} & alternative & & & \multirow{3}{*}{$\begin{array}{l}\mathrm{F}(1,28)=.24 \\
\mathrm{p}=.62\end{array}$} & $\mathrm{p}\left(\_\delta<<4.66 \_\right)$ \\
\hline & $\begin{array}{l}\text { visible } \\
\text { alternative values not }\end{array}$ & 15 & 8.4 & & $=.90$ \\
\hline & visible & 15 & 6.66 & & \\
\hline \multirow{3}{*}{$\begin{array}{l}\text { violations } \\
\text { of rule } 3\end{array}$} & alternative & & & \multirow{3}{*}{$\begin{array}{l}\mathrm{F}(1,28)=.007 \\
\mathrm{p}=.93\end{array}$} & $\mathrm{p}\left(\_\delta<<27.04 \_\right)$ \\
\hline & $\begin{array}{l}\text { visible } \\
\text { alternative values not }\end{array}$ & 15 & 7.93 & & $=.90$ \\
\hline & visible & 15 & 8.26 & & \\
\hline
\end{tabular}


Table 3.

Results of experiment $2 \mathrm{~b}$ : changing counter values (alternative values visible and alternative values not visible).

\begin{tabular}{|c|c|c|c|c|c|}
\hline $\begin{array}{l}\text { dependent } \\
\text { variable }\end{array}$ & condition & subjects & mean & $\begin{array}{l}\text { Memory load } \\
\text { hypothesis }\end{array}$ & $\begin{array}{l}\text { Bayesian } \\
\text { test }\end{array}$ \\
\hline $\begin{array}{l}\text { number of } \\
\text { moves }\end{array}$ & $\begin{array}{l}\text { alternative values } \\
\text { visible } \\
\text { alternative values not } \\
\text { visible }\end{array}$ & $\begin{array}{l}15 \\
15\end{array}$ & $\begin{array}{l}12.6 \\
15.87\end{array}$ & $\begin{array}{l}F(1,28)=2.04 \\
P=.16\end{array}$ & $\begin{array}{l}\mathrm{p}\left(\delta_{-} \_<6.14 \_\right) \\
=.90\end{array}$ \\
\hline solution time & $\begin{array}{l}\text { alternative values } \\
\text { visible } \\
\text { alternative values not } \\
\text { visible }\end{array}$ & $\begin{array}{l}15 \\
15\end{array}$ & $\begin{array}{l}3.31 \\
4.29 \\
\end{array}$ & $\begin{array}{l}\mathrm{F}(1,28)= \\
1.06 \\
\mathrm{p}=.31\end{array}$ & $\begin{array}{l}\mathrm{p}\left(\_\delta<\_2.30 \_\right) \\
=.90\end{array}$ \\
\hline $\begin{array}{l}\text { violations } \\
\text { of rule } 2\end{array}$ & $\begin{array}{l}\text { alternative values } \\
\text { visible } \\
\text { alternative values not } \\
\text { visible }\end{array}$ & $\begin{array}{l}15 \\
15\end{array}$ & $\begin{array}{l}2.53 \\
2.73\end{array}$ & $\begin{array}{l}F(1,28)= \\
.08 \\
p=.76\end{array}$ & $\begin{array}{l}\mathrm{p}\left(\delta_{-}<-1.20 \_\right) \\
=.90\end{array}$ \\
\hline $\begin{array}{l}\text { violations } \\
\text { rule } 3\end{array}$ & $\begin{array}{l}\text { alternative values } \\
\text { visible } \\
\text { alternative values not } \\
\text { visible }\end{array}$ & $\begin{array}{l}15 \\
15\end{array}$ & $\begin{array}{l}1.86 \\
2.60\end{array}$ & $\begin{array}{l}F(1,28)= \\
1.09 \\
p=.30\end{array}$ & $\begin{aligned} & \mathrm{p}\left(\delta_{-}<11.99 \_\right) \\
= & .90\end{aligned}$ \\
\hline
\end{tabular}


E. Clément and J.F. Richard

\section{Figure Caption}

Figure 1. The four problems used in experiment 1.

Figure 2. Average number of moves for the four problems (experiment 1).

Figure 3. Average solution time for the four problems (experiment 1).

Figure 4. Average number of violations of rule 2.

Figure 5. Average number of violations of rule 3 for each problem.

Figure 6. Mean relevancy index of the constraint prohibiting "to jump from one extreme state to the another" for each problem.

Figure 7. The Exchange problem used in experiment 2a.

Figure 8 . The counter problem used in experiment $2 \mathrm{~b}$. 